INTERNATIONAL JOURNAL OF ROBUST AND NONLINEAR CONTROL

Int. J. Robust Nonlinear Control 2009; 19:676-691

Published online 22 July 2008 in Wiley InterScience (www.interscience.wiley.com). DOI: 10.1002/rnc.1345

\title{
Observer design with guaranteed RMS gain for discrete-time LPV systems with Markovian jumps
}

\author{
Giuseppe C. Calafiore ${ }^{1, * \dagger}$ and Fabrizio Dabbene ${ }^{2}$ \\ ${ }^{1}$ Dipartimento di Automatica e Informatica, Politecnico di Torino, Torino, Italy \\ ${ }^{2}$ IEIIT-CNR, Politecnico di Torino, Torino, Italy
}

\begin{abstract}
SUMMARY
In this paper we consider the problem of designing state observers with guaranteed power-to-power (RMS) gain for a class of stochastic discrete-time linear systems that possess both measurable parameter variations and Markovian jumps in their dynamics. It is shown in the paper that an upper bound on the RMS gain of the observer can be characterized in terms of feasibility of a family of parameter-dependent linear matrix inequalities (LMIs). Any feasible solution to these LMIs can then be used to explicitly construct a parameter-varying jump observer that guarantees the desired performance level. This design framework is then specialized to a problem of state estimation for a linear parameter-varying plant whose state measurements are available through a lossy Bernoulli channel. Two numerical examples illustrate the results. Copyright (C) 2008 John Wiley \& Sons, Ltd.
\end{abstract}

Received 7 February 2008; Revised 3 April 2008; Accepted 20 April 2008

KEY WORDS: observers; LPV systems; Markov jump systems; estimation over lossy channels; linear matrix inequalities

\section{INTRODUCTION}

Linear parameter-varying (LPV) systems are commonly used to model dynamical systems that depend on a priori unknown, but online measurable time-varying parameters. These models have been extensively studied in the literature, mainly due to the reason that they provide a systematic way to design gain-scheduling filters and control laws for nonlinear systems; see, for instance, $[1,2]$. LPV approaches permit one to reduce the conservatism inherent in robust design methods, in cases where the a priori uncertain parameters can actually be acquired at the time of system

\footnotetext{
${ }^{*}$ Correspondence to: Giuseppe C. Calafiore, Dipartimento di Automatica e Informatica, Politecnico di Torino, Torino, Italy.

${ }^{\dagger}$ E-mail: giuseppe.calafiore@polito.it

Contract/grant sponsor: MIUR
}

Copyright (C) 2008 John Wiley \& Sons, Ltd. 
operation; see, e.g. [3-7]. There are indeed many relevant engineering applications where this is the case. Typical examples include, for instance, altitude, speed and pressure measurements in aerospace [8], asset and configuration in manufacturing and robotics [9], concentration and pressure measurements in chemical systems [10].

On a different direction, a considerable research effort has been recently devoted to the analysis of systems whose dynamics may change abruptly and in a random manner, and in particular to the class of Markov jump linear systems [11,12]. Such systems turn out to be particularly useful for modeling phenomena of control and estimation over networks, where random delays and packet losses may occur; see, for instance, the recent surveys $[13,14]$ and the references therein. The filtering problem for Markov jump systems has been extensively studied in the literature. For instance, de Souza and Fragoso $[15,16]$ treat the case of $H_{\infty}$ filtering in continuous and discrete time, when the Markov state (mode) is available to the filter, while Feng et al. [17] consider the mode-independent case. If uncertainty is present in the dynamics, a robust approach for filter design is considered in [18-20]. In a similar setting, Xiong and Lam [21] analyze the case when the Markov transition matrix is uncertain. Note that in a robust approach the filter is fixed once and for all, and it gives a priori guaranteed performance for all possible values of the uncertainty. Thus, the robust approach is certainly useful when no information about the parameters is available during operation, but may lead to conservative designs when the a priori parameter uncertainty is 'large'. However, as previously discussed, in many practical situations the a priori unknown parameters can actually be acquired online. This information can hence be exploited by the filter in order to 'adapt' to the changes in the system dynamics and improve performance. In this 'parameter varying' setup, the filter itself should therefore be allowed to vary with the parameters. Motivated by the above considerations, this paper explores the filter design problem for Markovian jump systems in the parameter-varying framework. To the best of the authors' knowledge, this setup has not been considered in the literature yet.

More precisely, in this note we study a filtering problem for linear discrete-time systems whose dynamic matrices jump according to a finite Markov chain and are functions of a priori unknown but measurable parameters (J-LPV systems). For this class of systems, we study the problem of designing a mode-dependent parameter-varying observer with guaranteed power-to-power (RMS) gain between the disturbance input and the estimation error.

The key result in Section 4 provides a condition for the RMS gain to be bounded, in terms of feasibility of a set of parameter-dependent linear matrix inequalities (LMIs). When these conditions are satisfied, a jump parameter-varying observer is also explicitly constructed. In Section 4.1, this result is specialized to the case of affine dependence of parameters ranging in a polytopic domain. In this case, the convex program induced by the LMI conditions can be solved efficiently.

Note that a parameter-varying plant that is to be observed or controlled through lossy channels can be suitably modeled by a system with both parameter variations and random jumps. Therefore, in Section 5 we specialize our results to parameter-varying plants whose output measurements are available through unreliable (Bernoulli) channels (i.e. with a certain probability measurements may not be available at some instants). Two numerical examples illustrate the proposed observer design technique.

\subsection{Notation}

$X^{\top}$ denotes the transpose of matrix $X ; X^{+}$denotes the Moore-Penrose pseudoinverse of $X$; and $X^{\perp}$ denotes an orthogonal complement of $X$, i.e. a matrix of maximum rank such that $X^{\perp} X=0$. 
$I_{n}$ denotes the $n \times n$ identity matrix, and $=0_{n, m}$ denotes an $n \times m$ matrix with zero entries; subscripts with dimensions are omitted when easily inferred from context. For $X_{1} \in \mathbb{R}^{n_{1}, n_{1}}, X_{2} \in \mathbb{R}^{n_{2}, n_{2}}$, $\operatorname{diag}\left(X_{1}, X_{2}\right)$ denotes the block diagonal matrix

$$
\left[\begin{array}{cc}
X_{1} & 0_{n_{1}, n_{2}} \\
0_{n_{2}, n_{1}} & X_{2}
\end{array}\right]
$$

$X \succ 0$ means that symmetric matrix $X$ is positive definite. We denote by $\operatorname{co}\left\{\theta_{1}, \ldots, \theta_{n}\right\}$ the convex hull of points $\theta_{1}, \ldots, \theta_{n}$. The space of finite power sequences is denoted by $\mathscr{U}$, that is, $\mathscr{U}$ is the space of sequences $z$ such that

$$
\|z\|_{\mathrm{rms}}^{2}=\lim _{T \rightarrow \infty} \frac{1}{T} \sum_{k=0}^{T} z_{k}^{\top} z_{k}<\infty
$$

Given a sequence of random variables $\left\{\xi_{1}, \ldots, \xi_{n}\right\}$ and a function $x\left(\xi_{1}, \ldots, \xi_{n}\right)$, we denote by $\mathbb{E}_{\left\{\xi_{1}, \ldots, \xi_{k}\right\}}[x]$ the expected value of $x$ computed with respect to $\left\{\xi_{1}, \ldots, \xi_{k}\right\}$, and by $\mathbb{E}_{\xi_{k+1}}\left[x \mid\left\{\xi_{1}, \ldots, \xi_{k}\right\}\right]$ the expectation of $x$ with respect to $\xi_{k+1}$, conditioned on $\left\{\xi_{1}, \ldots, \xi_{k}\right\}$. The $i$ th entry of vector $x$ is denoted by $x_{i}$ or $[x]_{i}$.

\section{PRELIMINARIES}

Consider a system $\mathscr{S}$ described by the following equations:

$$
\begin{aligned}
x_{k+1} & =A_{\xi_{k}}\left(\theta_{k}\right) x_{k}+B_{\xi_{k}}\left(\theta_{k}\right) u_{k} \\
y_{k} & =C_{\xi_{k}}\left(\theta_{k}\right) x_{k}+D_{\xi_{k}}\left(\theta_{k}\right) u_{k} \\
\theta_{k} & \in \Theta, \quad \xi_{k} \in\{1, \ldots, N\}
\end{aligned}
$$

where $x_{k} \in \mathbb{R}^{n}$ is the state at time $k ; u_{k} \in \mathbb{R}^{n_{u}}$ is a disturbance input at time $k ; \theta_{k}$ is a timevarying parameter that, a priori, is only known to belong to a given compact set $\Theta \subset \mathbb{R}^{n_{\theta}}$; $\xi_{k}$ is a homogeneous Markov chain taking values in the finite set $\{1, \ldots, N\}$; and $y_{k} \in \mathbb{R}^{n_{y}}$ is the stochastic output of the system. The Markov chain has given transition probabilities

$$
p_{i, j} \doteq \operatorname{prob}\left\{\xi_{k+1}=j \mid \xi_{k}=i\right\}, \quad i, j=1, \ldots, N
$$

The initial conditions for the system are specified by an initial state $x_{0}$ and mode $\xi_{0}$.

When useful for notational compactness, the system matrices are regrouped as

$$
S_{\xi_{k}}\left(\theta_{k}\right) \doteq\left[\begin{array}{cc}
A \xi_{k}\left(\theta_{k}\right) & B_{\xi_{k}}\left(\theta_{k}\right) \\
C_{\xi_{k}}\left(\theta_{k}\right) & D_{\xi_{k}}\left(\theta_{k}\right)
\end{array}\right]
$$

The following standard notion of stability is here adopted for the stochastic system $\mathscr{S}$ see, e.g. [22].

Definition 1 (Stochastic stability (SS))

Let $u_{k}=0$ for $k \geqslant 0$. System (1)-(3) is said to be stochastically stable if for any initial conditions $x_{0}, \xi_{0}$ it holds that $\sum_{k=0}^{\infty} \mathbb{E}_{\Xi_{k}}\left[x_{k}^{\top} x_{k}\right]<\infty$, where $\Xi_{k} \doteq\left\{\xi_{1}, \ldots, \xi_{k}\right\}$. 
For purely Markov jump systems (i.e. systems possessing only the Markov parameter $\xi_{k}$, without the time-varying term $\theta_{k}$ ), there exists a well-known necessary and sufficient characterization of stochastic stability; see, for instance, [11, 22, 23]. On the other hand, for purely parameter-varying systems (i.e. systems possessing only the $\theta_{k}$ parameter, without the Markovian jump structure), there exist several sufficient conditions for stability, the simplest of which (known as quadratic stability) is based on the existence of a common quadratic Lyapunov function for all possible values of $\theta_{k} \in \Theta$. The following lemma provides a sufficient condition for stochastic stability of the mixed parameter-varying and Markov jump system (1)-(3). This lemma extends to parameter-varying systems the stability results in $[11,22,23]$; a proof is provided in Appendix A.

\section{Lemma 1}

Suppose there exist matrices $P_{i} \succ 0, i=1, \ldots, N$, such that

$$
A_{i}^{\top}(\theta) \bar{P}_{i} A_{i}(\theta)-P_{i} \prec 0 \quad \forall \theta \in \Theta, \quad i=1, \ldots, N
$$

where $\bar{P}_{i} \doteq \sum_{j=1}^{N} p_{i, j} P_{j}$. Then, system $\mathscr{S}$ is stochastically stable.

\section{A BOUND ON RMS GAIN}

Assuming that $\mathscr{S}$ is stochastically stable, we say that the RMS gain of the system is less than $\gamma>0$ if

$$
\sup _{0 \neq u \in \mathscr{U}} \frac{\|y\|_{\mathrm{rms}}}{\|u\|_{\mathrm{rms}}}<\gamma
$$

for all $\xi_{0}$ and for all $y$ satisfying (1)-(3), with $x_{0}=0$. Here, the RMS value of the discrete-time stochastic signal $y$ is defined as $\|y\|_{\mathrm{rms}}{ }^{2} \doteq \lim _{T \rightarrow \infty}(1 / T) \sum_{k=0}^{T} \mathbb{E}_{\Xi_{k}}\left[y_{k}^{\top} y_{k}\right]$. The following lemma provides a sufficient condition for a finite upper bound on the RMS gain of system $\mathscr{S}$ to exist. A proof of this result is given in Appendix A.

Lemma 2

Suppose there exist symmetric matrices $P_{i} \succ 0, i=1, \ldots, N$ such that

$$
\left[\begin{array}{cc}
\operatorname{diag}\left(\bar{P}_{i}, I\right) & \operatorname{diag}\left(\bar{P}_{i}, I\right) S_{i}(\theta) \\
* & \operatorname{diag}\left(P_{i}, \gamma^{2} I\right)
\end{array}\right] \succ 0 \quad \forall \theta \in \Theta, \quad i=1, \ldots, N
$$

where $S_{i}(\theta)$ is defined in (4), and $\bar{P}_{i}$ are defined as in Lemma 1 . Then, system $\mathscr{S}$ is stochastically stable and has an RMS gain less than $\gamma$.

\section{Remark 3}

When $\Theta$ contains only one element, that is, $\theta_{k}$ is constant and fixed, condition (6) reduces to a known bounded real condition for Markovian jump systems, which has been proved to be both necessary and sufficient, under an additional hypothesis of weak controllability; see [24]. 


\section{RMS OBSERVER DESIGN}

Let system (1)-(3) be given. Following the standard approach for LPV systems, we assume that the time-varying parameter $\theta_{k}$ can be measured online at each time instant. We assume further that the current mode $\xi_{k}$ of the Markov chain is available at time $k$. This hypothesis is in agreement with most of the literature on Markovian jump systems see, e.g. [15, 16, 18-20]. On the basis of this information, we consider a filter having observer structure of the form

$$
\hat{x}_{k+1}=A_{\xi_{k}}\left(\theta_{k}\right) \hat{x}_{k}+L_{\xi_{k}}\left(\theta_{k}\right)\left(y_{k}-C_{\xi_{k}}\left(\theta_{k}\right) \hat{x}_{k}\right)
$$

where $L_{\xi_{k}}\left(\theta_{k}\right)$ is the parameter-varying filter gain, such that $L_{\xi_{k}}\left(\theta_{k}\right)=L_{i}\left(\theta_{k}\right)$ when the system is in mode $\xi_{k}=i$. The filtering error is defined as $e_{k} \doteq x_{k}-\hat{x}_{k}$. The filtering error system $\mathscr{F}$ having input $u$ and output $e$ is hence described by

$$
e_{k+1}=\mathscr{A}_{k} e_{k}+\mathscr{B}_{k} u_{k}
$$

with $\mathscr{A}_{k} \doteq A_{\xi_{k}}\left(\theta_{k}\right)-L_{\xi_{k}}\left(\theta_{k}\right) C_{\xi_{k}}\left(\theta_{k}\right)$ and $\mathscr{B}_{k} \doteq B_{\xi_{k}}\left(\theta_{k}\right)-L_{\xi_{k}}\left(\theta_{k}\right) D_{\xi_{k}}\left(\theta_{k}\right)$. In compact notation, the error system $\mathscr{F}$ is represented by the quadruple

$$
F_{\xi_{k}}\left(\theta_{k}\right) \doteq\left[\begin{array}{cc}
\mathscr{A}_{k} & \mathscr{B}_{k} \\
I_{n} & 0_{n, n_{u}}
\end{array}\right]=\left[\begin{array}{cc}
A_{\xi_{k}}\left(\theta_{k}\right) & B_{\xi_{k}}\left(\theta_{k}\right) \\
I_{n} & 0_{n, n_{u}}
\end{array}\right]+\left[\begin{array}{c}
-I_{n} \\
0_{n, n}
\end{array}\right] L_{\xi_{k}}\left(\theta_{k}\right)\left[C_{\xi_{k}}\left(\theta_{k}\right) D_{\xi_{k}}\left(\theta_{k}\right)\right]
$$

The following main theorem holds.

\section{Theorem 1}

Consider system $\mathscr{S}$ in (1)-(3), with $D_{i}(\theta)$ full row rank. Let (7) be an observer associated with system $\mathscr{S}$, and let $\gamma>0$ be given. Define

$$
\begin{aligned}
\bar{P}_{i} & \doteq \sum_{j=1}^{N} p_{i, j} P_{j}, \quad H_{i} \doteq\left(P_{i}-I_{n}\right)^{-1}, \quad R_{i}(\theta) \doteq D_{i}(\theta) D_{i}^{\top}(\theta) \\
N_{i}(\theta) & \doteq \text { an orthogonal basis for } \operatorname{ker} D_{i}(\theta): D_{i}(\theta) N_{i}(\theta)=0 \text { and } N_{i}^{\top}(\theta) N_{i}(\theta)=I_{n_{u}-n_{y}}
\end{aligned}
$$

If the following convex conditions in the variables $P_{i}=P_{i}^{\top}, i=1, \ldots, N$ :

$$
\left[\begin{array}{ccc|c}
\bar{P}_{i} & 0_{n, n} & \bar{P}_{i} A_{i}(\theta)-\bar{P}_{i} B_{i}(\theta) D_{i}^{\top}(\theta) R_{i}^{-1}(\theta) C_{i}(\theta) & \bar{P}_{i} B_{i}(\theta) N_{i}(\theta) \\
* & I_{n} & I_{n} & 0_{n, n_{u}-n_{y}} \\
* & * & P_{i}+\gamma^{2} C_{i}^{\top}(\theta) R_{i}^{-1}(\theta) C_{i}(\theta) & 0_{n, n_{u}-n_{y}} \\
\hline * & * & * & \gamma^{2} I_{n_{u}-n_{y}}
\end{array}\right] \succ 0
$$

are satisfied for all $\theta \in \Theta, i=1, \ldots, N$, then the observer gains

$$
L_{i}(\theta)=\left(A_{i}(\theta) H_{i} C_{i}^{\top}(\theta)+\frac{1}{\gamma^{2}} B_{i}(\theta) D_{i}^{\top}(\theta)\right)\left(C_{i}(\theta) H_{i} C_{i}^{\top}(\theta)+\frac{1}{\gamma^{2}} R_{i}(\theta)\right)^{-1}
$$


$i=1, \ldots, N$, guarantee that the filtering error system $\mathscr{F}$ in (9) is stochastically stable and has an RSM gain less than $\gamma$. Minimizing $\gamma^{2}$ subject to (10)-(11) then yields an optimized upper bound on the RMS gain of the filtering error system.

Proof

By Lemma 2, system $\mathscr{F}$ is stochastically stable and has an RMS gain less than $\gamma>0$ if there exist matrices $P_{i} \succ 0, i=1, \ldots, N$, such that for all $\theta \in \Theta, i=1, \ldots, N$

$$
\left[\begin{array}{cc}
\operatorname{diag}\left(\bar{P}_{i}, I_{n}\right) & \operatorname{diag}\left(\bar{P}_{i}, I_{n}\right) F_{i}(\theta) \\
* & \operatorname{diag}\left(P_{i}, \gamma^{2} I_{n_{u}}\right)
\end{array}\right] \succ 0
$$

Substituting (9) into (13), we explicitly obtain that the inequality

$$
Q_{i}(\theta)+U_{i} L_{i}(\theta) V_{i}^{\top}(\theta)+V_{i}(\theta) L_{i}^{\top}(\theta) U_{i}^{\top} \succ 0
$$

must hold $\forall \theta \in \Theta$, and for $i=1, \ldots, N$, with

$$
Q_{i}(\theta) \doteq\left[\begin{array}{cccc}
\bar{P}_{i} & 0_{n, n} & \bar{P}_{i} A_{i}(\theta) & \bar{P}_{i} B_{i}(\theta) \\
* & I_{n} & I_{n} & 0_{n, n_{u}} \\
* & * & P_{i} & 0_{n, n_{u}} \\
* & * & * & \gamma^{2} I_{n_{u}}
\end{array}\right], \quad U_{i} \doteq\left[\begin{array}{c}
-\bar{P}_{i} \\
0_{n, n} \\
0_{n, n} \\
0_{n_{u}, n}
\end{array}\right], \quad V_{i}(\theta) \doteq\left[\begin{array}{c}
0_{n, n_{y}} \\
0_{n, n_{y}} \\
C_{i}^{\top}(\theta) \\
D_{i}^{\top}(\theta)
\end{array}\right]
$$

Consider the following orthogonal complements of $U_{i}$ and $V_{i}(\theta)$, respectively:

$$
U^{\perp}=\left[\begin{array}{cccc}
0_{n} & I_{n} & 0_{n} & 0_{n, n_{u}} \\
0_{n} & 0_{n} & I_{n} & 0_{n, n_{u}} \\
0_{n_{u}, n} & 0_{n_{u}, n} & 0_{n_{u}, n} & I_{n_{u}}
\end{array}\right], \quad V_{i}^{\perp}(\theta)=\left[\begin{array}{cccc}
I_{n} & 0_{n} & 0_{n} & 0_{n, n_{u}} \\
0_{n} & I_{n} & 0_{n} & 0_{n, n_{u}} \\
0_{n} & 0_{n} & I_{n} & -C_{i}^{\top}(\theta) D_{i}^{\top+}(\theta) \\
0_{n_{v}, n} & 0_{n_{v}, n} & 0_{n_{v}, n} & N_{i}^{\top}(\theta)
\end{array}\right]
$$

Applying to (14) the elimination lemma (Lemma A1, in Appendix A), we obtain that (14) holds for suitable gains $L_{i}(\theta)$, if and only if $U_{i}^{\perp} Q_{i}(\theta) U_{i}^{\perp \top} \succ 0, V_{i}^{\perp}(\theta) Q_{i}(\theta) V_{i}^{\perp \top}(\theta) \succ 0$. After standard matrix manipulations, it can be verified that these two conditions are equivalent to (10), (11). Equation (12) then follows from (A5), with the position

$$
\begin{gathered}
\Upsilon_{1}=0_{n, n_{y},} \Upsilon_{2}=\left[\begin{array}{c}
0_{n, n_{y}} \\
C_{i}^{\top}(\theta) \\
D_{i}^{\top}(\theta)
\end{array}\right], \quad Z_{11}=\bar{P}_{i}^{-1}, \\
Z_{22}=\left[\begin{array}{ccc}
I_{n} & I_{n} & 0_{n, n_{u}} \\
* & P_{i} & 0_{n, n_{u}} \\
* & * & \gamma^{2} I_{n_{u}}
\end{array}\right], \quad Z_{12}^{\top}=-\left[\begin{array}{c}
0_{n, n} \\
A_{i}^{\top}(\theta) \\
B_{i}^{\top}(\theta)
\end{array}\right]
\end{gathered}
$$




\subsection{Polytopic parameter model}

Note that, in the frequently arising case when $\Theta$ contains infinite elements, conditions (11) in Theorem 1 require the satisfaction of infinitely many LMIs, i.e. the ensuing filter design problem amounts to solving a semi-infinite convex LMI problem. Such problems are typically encountered in the LPV approach to filtering and control and may be computationally difficult to solve. In order to address this issue, different approaches are generally followed. A first approach amounts to restricting the attention to a specific class of functions of the scheduling parameters. For example, one can assume that the matrices of the LPV model are expressed as a linear fractional transformation of the underlying parameters; see, for instance, $[4,5,25]$. The original problem is then relaxed to more tractable formulae that involve a finite number of LMIs. Another classical approach is to determine an approximate solution based on finite gridding of the set $\Theta$, see, for instance, $[3,5,7]$. Recently, approaches based on random gridding (sampling), which can deal with generic dependence on $\theta$, have been proposed in [26,27].

In this section we consider a special case in which the LMI conditions can be solved efficiently to any practical numerical accuracy. This special situation arises when $\Theta$ is a polytope (which encompasses the usual case of independent interval uncertainty), $A_{i}(\theta), B_{i}(\theta)$ are affine functions of $\theta$, and $C_{i}(\theta), D_{i}(\theta)$ do not depend on $\theta$. In this case, condition (11) is equivalent to a finite number of LMIs corresponding to the vertices of the polytope $\Theta$. This is formally stated in the following corollary.

Corollary 1

Consider system $\mathscr{S}$ in (1)-(3), and let $D_{\xi_{k}}\left(\theta_{k}\right)=D_{\xi_{k}}$ be full row rank, $C_{\xi_{k}}\left(\theta_{k}\right)=C_{\xi_{k}}$, and

$$
A_{\xi_{k}}\left(\theta_{k}\right)=A_{\xi_{k}}^{(0)}+\sum_{\ell=1}^{n_{\theta}}\left[\theta_{k}\right]_{\ell} A_{\xi_{k}}^{(\ell)}, \quad B_{\xi_{k}}\left(\theta_{k}\right)=B_{\xi_{k}}^{(0)}+\sum_{\ell=1}^{n_{\theta}}\left[\theta_{k}\right]_{\ell} B_{\xi_{k}}^{(\ell)}
$$

where

$$
\theta_{k} \in \Theta, \quad \Theta \doteq \operatorname{co}\left\{\theta^{(1)}, \ldots, \theta^{(m)}\right\}
$$

Let (7) be an observer associated with system $\mathscr{S}$, and $\gamma>0$ be given. Define $\bar{P}_{i}, H_{i}, R_{i}, N_{i}$ as in Theorem 1. If the following convex conditions in the variables $P_{i}=P_{i}^{\top}, i=1, \ldots, N$ :

$$
P_{i} \succ I_{n}, \quad\left[\begin{array}{ccc|c}
\bar{P}_{i} & 0_{n, n} & \bar{P}_{i} A_{i}\left(\theta^{(v)}\right)-\bar{P}_{i} B_{i}\left(\theta^{(v)}\right) D_{i}^{\top} R_{i}^{-1} C_{i} & \bar{P}_{i} B_{i}\left(\theta^{(v)}\right) N_{i} \\
* & I_{n} & I_{n} & 0_{n, n_{u}-n_{y}} \\
* & * & P_{i}+\gamma^{2} C_{i}^{\top} R_{i}^{-1} C_{i} & 0_{n, n_{u}-n_{y}} \\
\hline * & * & * & \gamma^{2} I_{n_{u}-n_{y}}
\end{array}\right] \succ 0
$$

are satisfied for $v=1, \ldots, m, i=1, \ldots, N$, then the observer gains

$$
L_{i}(\theta)=\left(A_{i}(\theta) H_{i} C_{i}^{\top}+\frac{1}{\gamma^{2}} B_{i}(\theta) D_{i}^{\top}\right)\left(C_{i} H_{i} C_{i}^{\top}+\frac{1}{\gamma^{2}} R_{i}\right)^{-1}
$$

$i=1, \ldots, N$, guarantee that the filtering error system $\mathscr{F}$ in (9) is stochastically stable and has an RMS gain less than $\gamma$. Minimizing $\gamma^{2}$ subject to (16) yields an optimized upper bound on the RMS gain of the error system $\mathscr{F}$. 


\subsection{Example 1}

To illustrate the result in Corollary 1, we considered the following numerical example with two Markovian states, i.e. $\xi_{k} \in\{1,2\}$, and two time-varying parameters, i.e. $\theta_{k} \in \mathbb{R}^{2}$. Specifically, we choose

$$
\begin{aligned}
A_{1}\left(\theta_{k}\right) & =\left[\begin{array}{cc}
-0.5 & -0.4 \\
0.1 & -0.8
\end{array}\right]+\left[\theta_{k}\right]_{1}\left[\begin{array}{ll}
1 & 0 \\
0 & 1
\end{array}\right]+\left[\theta_{k}\right]_{2}\left[\begin{array}{ll}
0 & 1 \\
1 & 0
\end{array}\right] \\
A_{2}\left(\theta_{k}\right) & =\left[\begin{array}{cc}
-0.75 & -0.5 \\
0.1 & -0.6
\end{array}\right]+\left[\theta_{k}\right]_{1}\left[\begin{array}{ll}
1 & 0 \\
0 & 1
\end{array}\right]+\left[\theta_{k}\right]_{2}\left[\begin{array}{ll}
0 & 1 \\
1 & 0
\end{array}\right] \\
B_{1}\left(\theta_{k}\right) & =\left[\begin{array}{ll}
0.1 & 0 \\
0.1 & 0
\end{array}\right]+\left[\theta_{k}\right]_{1}\left[\begin{array}{cc}
0.1 & 0 \\
0 & 0
\end{array}\right]+\left[\theta_{k}\right]_{2}\left[\begin{array}{ll}
0 & 0 \\
0.1 & 0
\end{array}\right] \\
B_{2}\left(\theta_{k}\right) & =B_{1}\left(\theta_{k}\right) \\
C_{1} & =\left[\begin{array}{ll}
-1.5 & 3
\end{array}\right] \\
C_{2} & =\left[\begin{array}{ll}
3 & -4
\end{array}\right] \\
D_{1} & =D_{2}=\left[\begin{array}{ll}
0 & 0.01
\end{array}\right]
\end{aligned}
$$

and suppose that the entries of $\theta_{k}$ are bounded in the interval $[-0.1,0.1]$, that is,

$$
\theta_{k} \in \operatorname{co}\left\{\left[\begin{array}{l}
-0.1 \\
-0.1
\end{array}\right],\left[\begin{array}{c}
-0.1 \\
0.1
\end{array}\right],\left[\begin{array}{c}
0.1 \\
-0.1
\end{array}\right],\left[\begin{array}{l}
0.1 \\
0.1
\end{array}\right]\right\}
$$

Moreover, the Markov transition probabilities are set to $p_{11}=0.9, p_{12}=0.1, p_{21}=0.7, p_{22}=0.3$. With these data, minimizing $\gamma^{2}$ subject to the conditions in (16) yielded optimal upper bound on the RMS gain of the filter $\gamma=0.2863$, and optimal parameter-varying observer gains

$$
\begin{aligned}
& L_{1}\left(\theta_{k}\right)=\left[\begin{array}{l}
-0.5707 \\
-0.4541
\end{array}\right]+\left[\theta_{k}\right]_{1}\left[\begin{array}{l}
0.6248 \\
0.6457
\end{array}\right]+\left[\theta_{k}\right]_{2}\left[\begin{array}{l}
0.6457 \\
0.6248
\end{array}\right] \\
& L_{2}\left(\theta_{k}\right)=\left[\begin{array}{l}
-0.4262 \\
-0.0215
\end{array}\right]+\left[\theta_{k}\right]_{1}\left[\begin{array}{l}
0.4899 \\
0.1175
\end{array}\right]+\left[\theta_{k}\right]_{2}\left[\begin{array}{l}
0.1175 \\
0.4899
\end{array}\right]
\end{aligned}
$$

A numerical simulation of the system (obtained for zero initial state conditions, normal random input with 0.01 variance and $\left.\left[\theta_{k}\right]_{1}=0.1 \sin (0.05 k),\left[\theta_{k}\right]_{2}=0.1 \cos (0.05 k)\right)$ yielded the trajectories shown in Figure 1. The experimental RMS filter gain resulting from this simulation was equal to 0.116 .

\section{OBSERVER DESIGN WITH MISSING MEASUREMENTS}

The developed framework for observer design has several applications. In particular, we next apply it in the context of systems with unreliable measurement channels, which is a key topic in networked control; see, e.g. the recent surveys [13, 14]. 

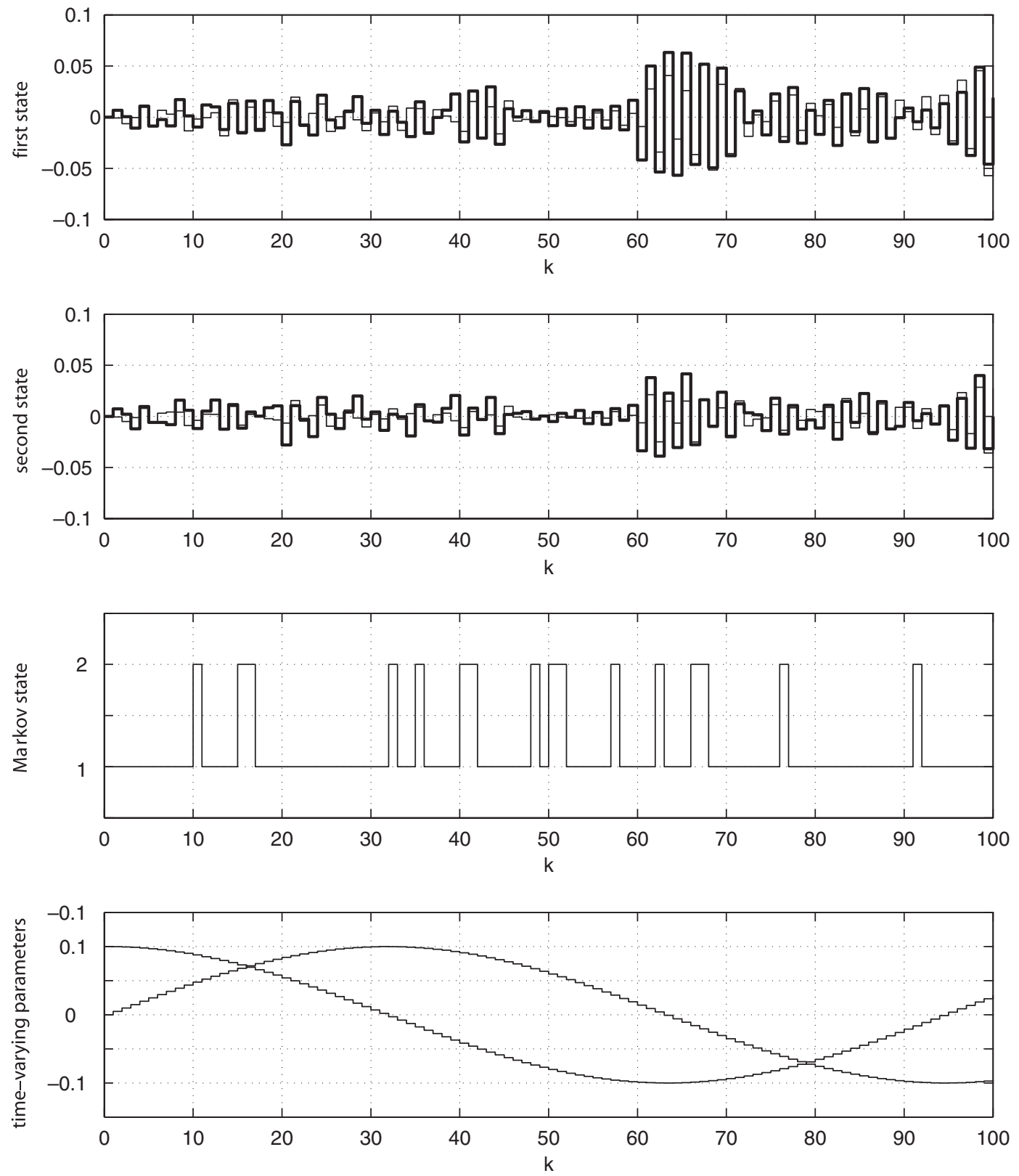

Figure 1. Simulation of system (bold) and estimator state trajectories for the system in Example 1. 
Assume that the plant is described by

$$
x_{k+1}=A\left(\theta_{k}\right) x_{k}+\tilde{B}\left(\theta_{k}\right) v_{k}
$$

and that measurements of the state are obtained through a Bernoulli channel, that is, with probability $p$ a measurement is available according to the equation $y_{k}=C x_{k}+\tilde{D} w_{k}$, and with probability $1-p$ the measure contains noise only, i.e. $y_{k}=\tilde{D} w_{k}$. This corresponds to a system having Markovian jumps in the $C$ matrix only:

$$
y_{k}=C_{\xi_{k}} x_{k}+\tilde{D} w_{k}
$$

with $\xi_{k} \in\{1,2\}$ and

$$
C_{\xi_{k}}= \begin{cases}C & \text { if } \xi_{k}=1 \\ 0 & \text { if } \xi_{k}=2\end{cases}
$$

The Markov chain governing the jump system is depicted in Figure 2.

This kind of lossy measurement models has been considered in several papers; see, for instance, $[13,28-30]$. In the context of this note, the above situation is captured simply by taking $u_{k}=$ $\left[v_{k}^{\top} w_{k}^{\top}\right]^{\top}, v_{k} \in \mathbb{R}^{n_{v}}, w_{k} \in \mathbb{R}^{n_{w}}$, and

$$
B_{\xi_{k}}\left(\theta_{k}\right)=\left[\begin{array}{ll}
\tilde{B}_{\xi_{k}}\left(\theta_{k}\right) & 0_{n, n_{w}}
\end{array}\right], \quad D_{\xi_{k}}\left(\theta_{k}\right)=\left[\begin{array}{ll}
0_{n_{y}, n_{v}} & \tilde{D}
\end{array}\right]
$$

The following corollary holds.

Corollary 2

Consider the LPV system (18) with unreliable measurement equation (19) governed by the Markovian model in Figure 2. Let $\tilde{D} \in \mathbb{R}^{n_{y}, n_{w}}$ be full row rank, $\Theta=\operatorname{co}\left\{\theta^{(1)}, \ldots, \theta^{(m)}\right\}$, and $A_{\xi_{k}}\left(\theta_{k}\right), B_{\xi_{k}}\left(\theta_{k}\right)$ be as in (15). Define

$$
\bar{P} \doteq \bar{P}_{1}=\bar{P}_{2}=p P_{1}+(1-p) P_{2}, \quad H \doteq\left(P_{1}-I_{n}\right)^{-1}
$$

If the following convex conditions in the symmetric matrix variables $P_{1}, P_{2}$ are satisfied for $v=1, \ldots, m$ :

$$
\begin{aligned}
& P_{1} \succ I_{n}, \quad P_{2} \succ I_{n} \\
& {\left[\begin{array}{cccc}
\bar{P} & 0_{n} & \bar{P} A\left(\theta^{(v)}\right) & \bar{P}\left[\tilde{B}\left(\theta^{(v)}\right) 0_{n, n_{w}-n_{y}}\right] \\
* & I_{n} & I_{n} & 0_{n, n_{u}-n_{y}} \\
* & * & P_{1}+\gamma^{2} C^{\top}\left[\tilde{D} \tilde{D}^{\top}\right]^{-1} C & 0_{n, n_{u}-n_{y}} \\
* & * & * & \gamma^{2} I_{n_{u}-n_{y}}
\end{array}\right] \succ 0} \\
& {\left[\begin{array}{cccc}
\bar{P} & 0_{n} & \bar{P} A\left(\theta^{(v)}\right) & \bar{P}\left[\tilde{B}\left(\theta^{(v)}\right) 0_{n, n_{w}-n_{y}}\right] \\
* & I_{n} & I_{n} & 0_{n, n_{u}-n_{y}} \\
* & * & P_{2} & 0_{n, n_{u}-n_{y}} \\
* & * & * & \gamma^{2} I_{n_{u}-n_{y}}
\end{array}\right] \succ 0}
\end{aligned}
$$




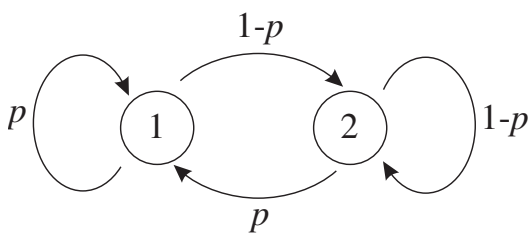

Figure 2. Markov chain for plants with missing measurements. State $\xi_{1}=1$ corresponds to an available measurement, and state $\xi_{2}=2$ corresponds to a missing measurement.

for $v=1, \ldots, m$. Then, the observer gains

$$
L_{i}(\theta)= \begin{cases}A(\theta) H C^{\top}\left(C H C^{\top}+\frac{1}{\gamma^{2}} \tilde{D} \tilde{D}^{\top}\right)^{-1} & \text { for } i=1 \\ 0 & \text { for } i=2\end{cases}
$$

guarantee that the filtering error system $\mathscr{F}$ in (9) is stochastically stable and has an RMS gain less than $\gamma$. Minimizing $\gamma^{2}$ subject to (22)-(23) yields an optimized upper bound on the RMS gain of the error system $\mathscr{F}$.

Proof

A proof is obtained by applying Corollary 1 to this particular special case. More precisely, define

$$
\tilde{N} \in \mathbb{R}^{n_{w}, n_{w}-n_{y}} \doteq \text { an orthogonal basis for } \operatorname{ker} \tilde{D}: \tilde{D} \tilde{N}=0 \quad \text { and } \quad \tilde{N}^{\top} \tilde{N}=I_{n_{w}-n_{y}}
$$

Then, the orthogonal complements $N_{i}$ defined in Corollary 1 are given by $N_{1}=N_{2}=\operatorname{diag}(I, \tilde{N})$. Hence, using the positions in (20), (21), with $C_{1}=C, C_{2}=0$, and substituting these data into (16), (17), we obtain the statement.

The previous result for the observer gain structure is in agreement with intuition. That is, when no measurement is available, the observer gain is zero, and the filter simply propagates forward the plant dynamics.

\subsection{Example 2}

We adapt an example originally considered in [29] in the context of Kalman filtering with intermittent observations. Consider an LPV system of the form

$$
x_{k+1}=\left[\begin{array}{ccc}
1.25 & 1 & 0 \\
0 & 0.9 & 0 \\
0 & 0 & 0.6+\theta
\end{array}\right] x_{k}+\sqrt{20} v_{k}
$$

with $v_{k} \in \mathbb{R}^{3}$. The state matrix $A(\theta)$ depends on a time-varying parameter $\theta$, which is supposed to be measurable online and to be bounded in the polytope $\Theta=\{0, \rho\}$. When $\rho=0$ we recover the nonvarying case considered in [29]. The state of the system is measured through a Bernoulli channel, 
Table I. Value of $p_{\text {lim }}$ for different values of $\rho$.

\begin{tabular}{lllllll}
\hline$\rho$ & 0 & 0.25 & 0.35 & 0.4 & 0.45 & 0.5 \\
$p_{\text {lim }}$ & 0.361 & 0.361 & 0.361 & 0.369 & 0.591 & 0.839 \\
\hline
\end{tabular}

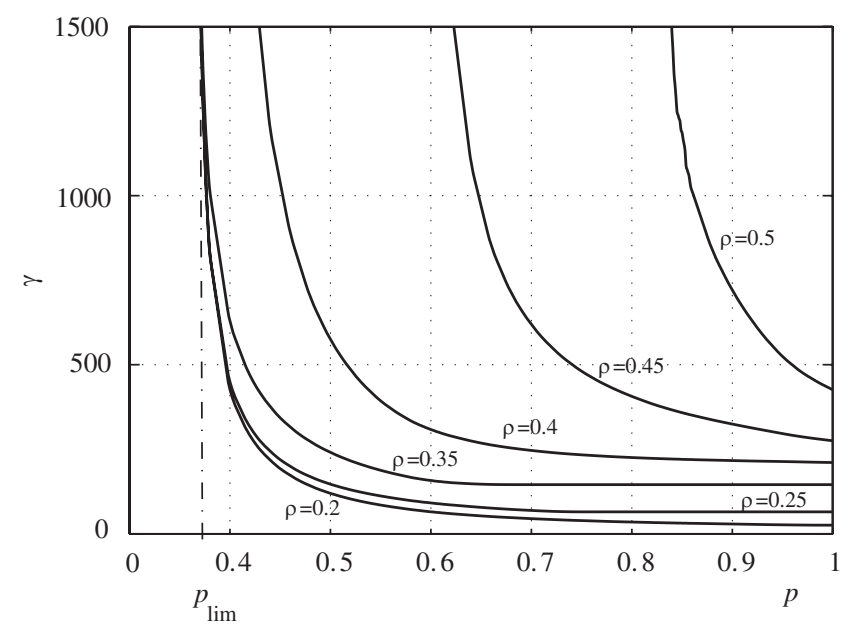

Figure 3. Plot of the RMS gain versus the probability of receiving a good measurement, for different values of the radius of parameter variation $\rho$.

with a probability of missing measurement equal to $1-p$. In the case of available measurement, the output equation is given by

$$
y_{k}=\left[\begin{array}{lll}
1 & 0 & 2
\end{array}\right] x_{k}+\sqrt{2.5} w_{k}
$$

with $w_{k} \in \mathbb{R}$. Note that, since the considered system is unstable, we can expect that if the channel is unreliable (i.e. $p$ is 'small') the observer will not be able to correctly follow the state trajectory, whereas if measurements arrive frequently (i.e. $p$ is 'large') the filtering error shall be stable. It would therefore be interesting to determine the threshold value of measurement rate $p$ below which it is no longer possible to correctly estimate the states of the system. Specifically, we can compute an upper bound $p_{\text {lim }}$ on the minimum value of $p$ such that the filtering error system $\mathscr{F}$ is stable. That is, for fixed $\rho$, we consider the minimization problem

$$
\underset{\gamma^{2}, p, P_{1}, P_{2}}{\operatorname{minimize}} p \quad \text { subject to } p \in(0,1) \text { and }(22)-(23)
$$

This problem can be easily solved by bisection over $p$, where each step in the bisection method requires checking feasibility of the LMIs considered in Corollary 2. For $\rho=0$, we obtain the value of $p_{\text {lim }}=0.361$, which numerically coincides with the one obtained in [29]. Then, we run the optimization (24) for increasing values of the uncertainty radius $\rho$. The results are reported in Table I.

In Figure 3 we also report the values of the RMS gain $\gamma$ obtained optimizing $\gamma^{2}$ over (22)-(23) for different values of $\rho$ and values of $p$ ranging in $\left(p_{\lim }, 1\right)$. 
We note, as expected, that an increase in the radius of uncertainty $\rho$ corresponds to a degradation in the performance of the filter. Moreover, in our experiments, we observed that an abrupt change in the limit value in the admissible level of measurement rate $p_{\text {lim }}$ occurs when $\rho$ reaches the value 0.4. This type of behavior has also been observed in [29] and corresponds to the situation where the system matrix $A(\theta)$ may have more than one unstable eigenvalue.

\section{CONCLUSIONS}

This paper discussed an RMS filtering problem for discrete-time systems that present both parameter variations and Markovian jumps in their system matrices. The key result in Theorem 1 provides LMI conditions for guaranteeing that the filtering error system is stable and has (squared) RMS gain less than a given level $\gamma^{2}$. An optimized filter can then be obtained by minimizing the level $\gamma^{2}$ subject to these conditions. The resulting convex optimization problem can be solved exactly when $\Theta$ is of finite cardinality, or approximately via deterministic or probabilistic gridding techniques, otherwise. In the particular case of polytopic LPV parameters, the problem becomes a standard convex LMI optimization problem, which can be solved in polynomial time. Models of LPV systems whose measurements are available through lossy channels fit into the considered class and have been analyzed as a special case in Section 5.

\section{APPENDIX A}

\section{Proof of Lemma 1}

We start with a preliminary technical result: System $\mathscr{S}$ is stochastically stable if there exists a stochastic Lyapunov function $V(k) \doteq V\left(x_{k}, \xi_{k}\right)=x_{k}^{\top} P_{\xi_{k}} x_{k}$, with $P_{\xi_{k}}=P_{i} \succ 0$ when $\xi_{k}=i$, such that for all $x_{k}$ satisfying the system equations (1)-(3) it holds that $\mathbb{E}_{\Xi_{k+1}}[\Delta V(k)]<0$, where $\Delta V(k) \doteq$ $V(k+1)-V(k)$.

To prove this statement, note that, since $\mathbb{E}_{\Xi_{k+1}}[\Delta V(k)]<0$ and $V(k)>0$, there exists $\varepsilon \in$ $(0,1)$ such that $\mathbb{E}_{\Xi_{k+1}}[\Delta V(k)]=\mathbb{E}_{\Xi_{k+1}}[V(k+1)]-\mathbb{E}_{\Xi_{k}}[V(k)] \leqslant-\varepsilon \mathbb{E}_{\Xi_{k}}[V(k)]$; hence, $\mathbb{E}_{\Xi_{k+1}}[V(k+$ $1)] \leqslant(1-\varepsilon) \mathbb{E}_{\Xi_{k}}[V(k)]$. Therefore, $\mathbb{E}_{\Xi_{k}}[V(k)] \leqslant(1-\varepsilon)^{k} V(0)$. Summing over $k$ from 0 to infinity, we get $\sum_{k=0}^{\infty} \mathbb{E}_{\Xi_{k}}[V(k)]=\sum_{k=0}^{\infty} \mathbb{E}_{\Xi_{k}}\left[x_{k}^{\top} P_{\xi_{k}} x_{k}\right] \leqslant(1 / \varepsilon) V(0)$. Since $P_{\xi_{k}} \succ 0$, this implies that $\sum_{k=0}^{\infty} \mathbb{E}_{\Xi_{k}}\left[x_{k}^{\top} x_{k}\right]<\infty$, which proves the preliminary statement.

Suppose now (5) holds and define the stochastic Lyapunov function $V(k)=x_{k}^{\top} P_{\xi_{k}} x_{k}$ with $P_{\xi_{k}}=$ $P_{i}$, for $\xi_{k}=i$. Then, we have $\mathbb{E}_{\Xi_{k+1}}[\Delta V(k)]=\mathbb{E}_{\Xi_{k}}\left[\mathbb{E}_{\xi_{k+1}}\left[\Delta V(k) \mid \Xi_{k}\right]\right]=\mathbb{E}_{\Xi_{k}}\left[\mathbb{E}_{\xi_{k+1}}\left[V(k+1) \mid \Xi_{k}\right]-\right.$ $V(k)]$, where the last equality follows from the fact that $V(k)$ does not depend on $\xi_{k+1}$. Note now that $\mathbb{E}_{\xi_{k+1}}\left[V(k+1) \mid \Xi_{k}\right]=\mathbb{E}_{\xi_{k+1}}\left[x_{k+1}^{\top} P_{\xi_{k+1}} x_{k+1} \mid \Xi_{k}\right]=\mathbb{E}_{\xi_{k+1}}\left[x_{k}^{\top} A_{\xi_{k}}\left(\theta_{k}\right)^{\top} P_{\xi_{k+1}} A_{\xi_{k}}\left(\theta_{k}\right) x_{k} \mid \Xi_{k}\right]=$ $x_{k}^{\top} A_{\xi_{k}}\left(\theta_{k}\right) \mathbb{E}_{\xi_{k+1}}\left[P_{\xi_{k+1}} \mid \Xi_{k}\right] A_{\xi_{k}}\left(\theta_{k}\right) x_{k}$. From the Markov property, it then follows that

$$
\mathbb{E}_{\xi_{k+1}}\left[P_{\xi_{k+1}} \mid \Xi_{k}\right]=\mathbb{E}_{\xi_{k+1}}\left[P_{\xi_{k+1}} \mid \xi_{k}\right]=\sum_{j=1}^{N} p_{\xi_{k}, j} P_{\xi_{k}}=\bar{P}_{\xi_{k}}
$$

hence $\mathbb{E}_{\Xi_{k+1}}[\Delta V(k)]=\mathbb{E}_{\Xi_{k}}\left[x_{k}^{\top}\left(A_{\xi_{k}}\left(\theta_{k}\right)^{\top} \bar{P}_{\xi_{k}} A_{\xi_{k}}\left(\theta_{k}\right)-P_{\xi_{k}}\right) x_{k}\right]$. It follows from (5) that this latter expression is negative for all $x_{k}$ satisfying the system equations; therefore, stochastic stability of $\mathscr{S}$ follows from the preliminary result. 
Proof of Lemma 2

Again, we start by stating a preliminary result: Suppose there exists a stochastic Lyapunov function $V(k)=V\left(x_{k}, \xi_{k}\right)=x_{k}^{\top} P_{\xi_{k}} x_{k}$, with $P_{\xi_{k}}=P_{i} \succ 0$ for $\xi_{k}=i$, such that

$$
\mathbb{E}_{\Xi_{k+1}}[\Delta V(k)]<\gamma^{2} u_{k}^{\top} u_{k}-\mathbb{E}_{\Xi_{k}}\left[y_{k}^{\top} y_{k}\right]
$$

holds for all $u \in \mathscr{U}$, for all $x_{k}, y_{k}$ satisfying (1)-(3), and for all $k \geqslant 0$. Then, system $\mathscr{S}$ is stochastically stable and has an RMS gain less than $\gamma>0$.

To prove this preliminary statement, note that since (A1) must hold for all $u \in \mathscr{U}$, choosing $u=0$, we have $\mathbb{E}_{\Xi_{k+1}}[\Delta V(k)]<0$, which implies stochastic stability. Note next that

$$
\sum_{k=0}^{T} \mathbb{E}_{\Xi_{k+1}}[\Delta V(k)]=\mathbb{E}_{\Xi_{T+1}}[V(T+1)]-V(0)=\mathbb{E}_{\Xi_{T+1}}[V(T+1)]
$$

where we used the fact that $x_{0}=0$ when $V(0)=0$. Therefore, summing over (A1) for $k=0$ to $T$, we get $\mathbb{E}_{\Xi_{T+1}}[V(T+1)]<\gamma^{2} \sum_{k=0}^{T} u_{k}^{\top} u_{k}-\sum_{k=0}^{T} \mathbb{E}_{\Xi_{k}}\left[y_{k}^{\top} y_{k}\right], \forall T \geqslant 0$. Since $\mathbb{E}_{\Xi_{T+1}}[V(T+1)] \geqslant 0$, it follows that $\sum_{k=0}^{T} \mathbb{E}_{\Xi_{k}}\left[y_{k}^{\top} y_{k}\right]<\gamma^{2} \sum_{k=0}^{T} u_{k}^{\top} u_{k}, \forall T \geqslant 0$. Hence, dividing both sides by $T$ and taking the limit for $T \rightarrow \infty$, we get that $\|y\|_{\mathrm{rms}}{ }^{2}<\gamma^{2}\|u\|_{\mathrm{rms}}{ }^{2}$ holds for all $u \in \mathscr{U}$ and $y_{k}$ satisfying (1)-(3), which proves the preliminary statement.

Suppose now that (6) holds and define $V(k)=x_{k}^{\top} P_{\xi_{k}} x_{k}$ with $P_{\xi_{k}}=P_{i}$ for $\xi_{k}=i$. We have

$$
\begin{aligned}
\mathbb{E}_{\Xi_{k}} & {\left[\mathbb{E}_{\xi_{k+1}}\left[V(k+1) \mid \Xi_{k}\right]-V(k)\right] } \\
& =\mathbb{E}_{\Xi_{k}}\left[\mathbb{E}_{\xi_{k+1}}\left[\left(A_{\xi_{k}}\left(\theta_{k}\right) x_{k}+B_{\xi_{k}}\left(\theta_{k}\right) u_{k}\right)^{\top} P_{\xi_{k+1}}\left(A_{\xi_{k}}\left(\theta_{k}\right) x_{k}+B_{\xi_{k}}\left(\theta_{k}\right) u_{k}\right) \mid \Xi_{k}\right]-x_{k}^{\top} P_{\xi_{k}} x_{k}\right] \\
& =\mathbb{E}_{\Xi_{k}}\left[\left(A_{\xi_{k}}\left(\theta_{k}\right) x_{k}+B_{\xi_{k}}\left(\theta_{k}\right) u_{k}\right)^{\top} \bar{P}_{\xi_{k}}\left(A_{\xi_{k}}\left(\theta_{k}\right) x_{k}+B_{\xi_{k}}\left(\theta_{k}\right) u_{k}\right)-x_{k}^{\top} P_{\xi_{k}} x_{k}\right] \\
& <\gamma^{2} u_{k}^{\top} u_{k}-\mathbb{E}_{\Xi_{k}}\left[\left(C_{\xi_{k}}\left(\theta_{k}\right) x_{k}+D_{\xi_{k}}\left(\theta_{k}\right) u_{k}\right)^{\top}\left(C_{\xi_{k}}\left(\theta_{k}\right) x_{k}+D_{\xi_{k}}\left(\theta_{k}\right) u_{k}\right)\right]
\end{aligned}
$$

The latter expression may be rewritten as

$\mathbb{E}_{\Xi_{k}}\left[\begin{array}{c}x_{k} \\ u_{k}\end{array}\right]^{\top}\left[\begin{array}{cc}A_{\xi_{k}}\left(\theta_{k}\right)^{\top} \bar{P}_{\xi_{k}} A_{\xi_{k}}\left(\theta_{k}\right)-P_{\xi_{k}}+C_{\xi_{k}}\left(\theta_{k}\right)^{\top} C_{\xi_{k}}\left(\theta_{k}\right) & A_{\xi_{k}}\left(\theta_{k}\right)^{\top} \bar{P}_{\xi_{k}} B_{\xi_{k}}\left(\theta_{k}\right)+C_{\xi_{k}}\left(\theta_{k}\right)^{\top} D_{\xi_{k}}\left(\theta_{k}\right) \\ * & B_{\xi_{k}}\left(\theta_{k}\right)^{\top} \bar{P}_{\xi_{k}} B_{\xi_{k}}\left(\theta_{k}\right)+D_{\xi_{k}}\left(\theta_{k}\right)^{\top} D_{\xi_{k}}\left(\theta_{k}\right)-\gamma^{2} I\end{array}\right]\left[\begin{array}{c}x_{k} \\ u_{k}\end{array}\right]<0$

which is satisfied for all $x_{k}, u_{k}$ that satisfy the system equations if the following LMI holds for all $\theta \in \Theta, i=1, \ldots, N$ :

$$
\left[\begin{array}{cc}
-A_{i}^{\top}(\theta) \bar{P}_{i} A_{i}(\theta)+P_{i}-C_{i}^{\top}(\theta) C_{i}(\theta) & -A_{i}^{\top}(\theta) \bar{P}_{i} B_{i}(\theta)-C_{i}^{\top}(\theta) D_{i}(\theta) \\
* & -B_{i}^{\top}(\theta) \bar{P}_{i} B_{i}(\theta)-D_{i}^{\top}(\theta) D_{i}(\theta)+\gamma^{2} I
\end{array}\right] \succ 0
$$

Let $\Omega_{i} \doteq \operatorname{diag}\left(P_{i}, \gamma^{2} I\right), \bar{\Omega}_{i} \doteq \operatorname{diag}\left(\bar{P}_{i}, I\right)$ and

$$
S_{i}(\theta) \doteq\left[\begin{array}{ll}
A_{i}(\theta) & B_{i}(\theta) \\
C_{i}(\theta) & D_{i}(\theta)
\end{array}\right]
$$

Then the left-hand side of the previous inequality is rewritten as

$$
\Omega_{i}-S_{i}^{\top}(\theta) \bar{\Omega}_{i} S_{i}(\theta)=\Omega_{i}-S_{i}^{\top}(\theta) \bar{\Omega}_{i} \bar{\Omega}_{i}^{-1} \bar{\Omega}_{i} S_{i}(\theta)
$$


Hence, applying the Schur complement rule to the inequality, we obtain

$$
\left[\begin{array}{cc}
\bar{\Omega}_{i} & \bar{\Omega}_{i} S_{i}(\theta) \\
S_{i}^{\top}(\theta) \bar{\Omega}_{i} & \Omega_{i}
\end{array}\right] \succ 0
$$

which is the statement in (6).

Lemma A1 (Elimination, see [31])

The matrix inequality

$$
Q+U Y V^{\top}+V Y^{\top} U^{\top}>0
$$

holds for some $Y$ if and only if

$$
\begin{array}{llll}
U^{\perp} Q U^{\perp \top} \succ 0 & \text { or } & U U^{\top} \succ 0 \\
V^{\perp} Q V^{\perp \top} \succ 0 & \text { or } & V V^{\top} \succ 0
\end{array}
$$

where $U^{\perp}, V^{\perp}$ are the orthogonal complements of $U, V$, respectively. Furthermore, if $U, V$ are full column rank and (A3), (A4) are satisfied, then a matrix $Y$ that satisfies (A2) is given by

$$
Y=\left(\Upsilon_{1}-Z_{12} Z_{22}^{-1} \Upsilon_{2}\right)\left(\Upsilon_{2}^{\top} Z_{22}^{-1} \Upsilon_{2}\right)^{-1}
$$

where

$$
\left[\begin{array}{c}
\Upsilon_{1} \\
\Upsilon_{2}
\end{array}\right] \doteq\left[\begin{array}{l}
U^{+} \\
U^{\perp}
\end{array}\right] V, \quad\left[\begin{array}{ll}
Z_{11} & Z_{12} \\
Z_{12}^{\top} & Z_{22}
\end{array}\right] \doteq\left[\begin{array}{c}
U^{+} \\
U^{\perp}
\end{array}\right] Q\left[\begin{array}{c}
U^{+} \\
U^{\perp}
\end{array}\right]^{\top}
$$

\section{ACKNOWLEDGEMENTS}

This work is supported by MIUR under the FIRB project 'Learning, randomization and guaranteed predictive inference for complex uncertain systems'.

\section{REFERENCES}

1. Leith DJ, Leithead WE. Survey of gain-scheduling analysis and design. International Journal of Control 2000; 73(11):1001-1025.

2. Rugh WJ, Shamma JS. Research on gain-scheduling. Automatica 2000; 36(10):1401-1425.

3. Apkarian P, Adams RJ. Advanced gain-scheduling techniques for uncertain systems. IEEE Transactions on Control Systems and Technology 1998; 6(1):21-32.

4. Apkarian P, Gahinet P. A convex characterization of gain-scheduled $H_{\infty}$ controllers. IEEE Transactions on Automatic Control 1995; 40(5):853-864.

5. Becker G, Packard A. Robust performance of linear parametrically varying systems using parametrically-dependent linear feedback. Systems and Control Letters 1994; 23(3):205-215.

6. Scherer CW. LPV control and full block multipliers. Automatica 2001; 37(3):361-375.

7. Wu F, Grigoriadis KM. LPV systems with parameter-varying time delays: analysis and control. Automatica 2001; 37(2):221-229.

8. Szászi I, Marcos A, Balas G, Bokor J. Linear parameter-varying detection filter design for a Boeing 747-100/200 aircraft. Journal of Guidance, Control, and Dynamics 2005; 28(3):461-470.

9. Yu Z, Chen H, Woo P. Gain scheduled LPV $H_{\infty}$ control based on LMI approach for a robotic manipulator. Journal of Robotic Systems 2002; 19(12):585-593. 
10. Kothare MV, Mettler B, Morari M, Bendotti P, Falinower C-M. Linear parameter varying model predictive control for steam generator level control. Computers and Chemical Engineering 1997; 21(1):861-866.

11. Boukas EK, Yang H. Stability of stochastic systems with jumps. Mathematical Problems in Engineering 1996; 3(2):173-185.

12. Costa OLV, Fragoso MD, Marques RP. Discrete-time Markov Jump Linear Systems. Probability and its Applications. Springer: New York, 2005.

13. Hespanha J, Naghshtabrizi P, Xu Y. A survey on recent results in networked control systems. Proceedings of the IEEE (Special Issue on Technology of Networked Control Systems) 2007; 95(1):138-162.

14. Schenato L, Sinopoli B, Franceschetti M, Poolla K, Sastry SS. Foundations 1 of control and estimation over lossy networks. Proceedings of the IEEE (Special Issue on Networked Control Systems) 2007; 95(1):163-187.

15. de Souza CE, Fragoso MD. $H_{\infty}$ filtering for Markovian jump linear systems. International Journal of Systems Science 2002; 33(11):909-915.

16. de Souza CE, Fragoso MD. $H_{\infty}$ filtering for discrete time linear systems with Markovian jumping parameters. Journal of Robust and Nonlinear Control 2003; 13(14):1299-1316.

17. de Souza CE, Trofino A, Barbosa KA. Mode-independent $H_{\infty}$ filters for Markovian jump linear systems. IEEE Transactions on Automatic Control 2006; 51(11):1837-1841.

18. Boukas EK, Liu ZK. Robust $H_{\infty}$ filtering for polytopic uncertain time-delay systems with Markov jumps. Computers and Electrical Engineering 2002; 28(3):171-193.

19. Shi P, Mahmoud M, Nguang SK. Robust filtering for jumping systems with mode-dependent delays. Signal Processing 2006; 86(1):140-152.

20. Xu SY, Chen TW, Lam J. Robust $H_{\infty}$ filtering for uncertain Markovian jump systems with mode-dependent time delays. IEEE Transactions on Automatic Control 2003; 48(5):900-907.

21. Xiong JL, Lam J. Fixed-order robust $H_{\infty}$ filter design for Markovian jump systems with uncertain switching probabilities. IEEE Transactions on Signal Processing 2006; 54(4):1421-1430.

22. Feng X, Loparo KA, Ji Y, Chizeck HJ. Stochastic stability properties of jump linear systems. IEEE Transactions on Automatic Control 1992; AC-37(1):38-53.

23. Costa OLV, Fragoso MD. Stability results for discrete-time linear-systems with Markovian jumping parameters. Journal of Mathematical Analysis and Applications 1993; 179(1):154-178.

24. Seiler P, Sengupta R. A bounded real lemma for jump systems. IEEE Transactions on Automatic Control 1996; AC-48(9):1651-1654.

25. Köse ǏE, Jabbari F, Schmitendorf WE. A direct characterization of $\mathscr{L}_{2}$-gain controllers for LPV systems. IEEE Transactions on Automatic Control 1998; 43(9):1302-1307.

26. Calafiore G, Campi MC. The scenario approach to robust control design. IEEE Transactions on Automatic Control 2006; 51(5):742-753.

27. Fujisaki Y, Dabbene F, Tempo R. Probabilistic robust design of LPV control systems. Automatica 2003; 39(8): 1323-1337.

28. Fletcher AK, Rangan S, Goyal VK. Estimation from lossy sensor data: jump linear modeling and Kalman filtering. Information Processing in Sensor Networks, Berkeley, CA, 2004; 251-258.

29. Sinopoli B, Schenato L, Franceschetti M, Poolla K, Jordan MI, Sastry SS. Kalman filtering with intermittent observations. IEEE Transactions on Automatic Control 2004; 49(9):1453-1464.

30. Craig Smith S, Seiler P. Optimal pseudo-steady-state estimators for systems with Markovian intermittent measurement. Proceedings of the American Control Conference, 2002; 3021-3027.

31. Skelton RE, Iwasaki T, Grigoriadis KM. A Unified Algebraic Approach to Linear Control Design. Taylor \& Francis: London, 1998. 